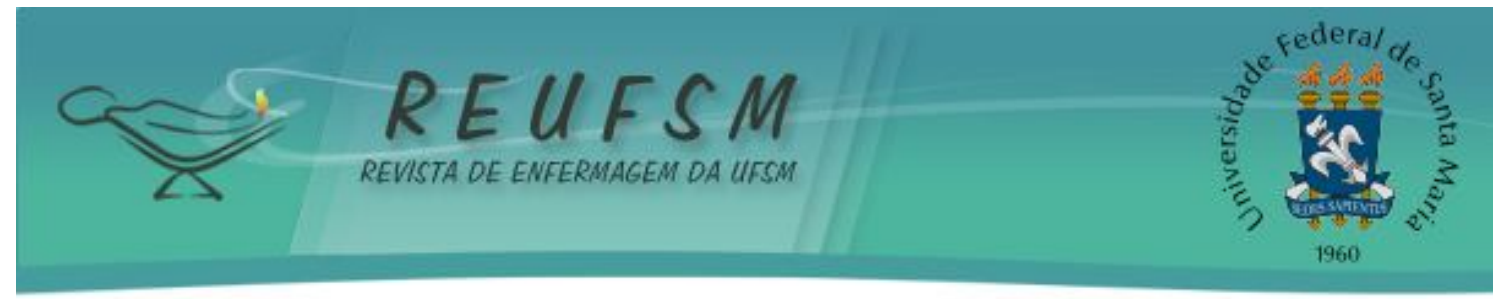

ARTIGO ORIGINAL

\title{
MORTALIDADE INFANTIL SEGUNDO CARACTERÍSTICAS DA MÃE E GESTAÇÃO NA CIDADE DE SANTA MARIA, RS
}

\section{INFANT MORTALITY BY MOTHER'S CHARACTERISTICS AND PREGNANCY IN THE CITY OF SANT A MARIA, RS}

\section{MORT ALIDAD INFANT IL SE GÚN CARACTERÍST ICAS DE LA MADRE Y EL EMBARAZO EN LA CIUDAD DE SANT A MARIA, RS}

\author{
Tassiana Potrich ${ }^{1}$ \\ Laissa Benites Medeiros ${ }^{2}$ \\ Raquel Possobon ${ }^{3}$ \\ Patrícia Vianna ${ }^{4}$ \\ Ruth Maurer da Silva ${ }^{5}$ \\ Eliane Tatsch Neves ${ }^{6}$
}

RESUMO: Objetivo: analisar a mortalidade infantil segundo características da mãe, gestação e peso ao nascer na cidade de Santa Maria, RS. Métodos: pesquisa quantitativa descritiva. As informações foram coletadas no site do Ministério da Saúde, no banco de dados do Departamento de Informática do Sistema Único de Saúde-DATASUS, no período entre 2000 a 2008. Resultados: apontaram maior prevalência de mortalidade infantil em crianças com peso ao nascer, entre 500 e 999g, sendo que as mães possuíam escolaridade abaixo dos sete anos e com idade entre 15 e 24 anos. Em relação à gestação, o período em que ocorreu o maior número de óbitos foi entre 32 e 41 semanas, na ocorrência de parto cesáreo. Considerações finais: recomendam-se novos estudos relacionando os achados com outros fatores de risco, como realização ou não de consultas pré-natal, alimentação materna, número de gestações, para assim determinar um diagnóstico fidedigno da mortalidade infantil do município.

Descritores: Mortalidade infantil; Gestação; Peso ao nascer.

ABSTRACT: Objective: To examine infant mortality according to characteristics of the mother, pregnancy and birth weight in the city of Santa Maria, RS. Methods: A descriptive quantitative research. Information was collected on the website of the Ministry of Health, the database of the Department of the Unified Health System-DATASUS in the period 2000 to 2008. Results: it showed a higher prevalence of mortality in children with birth weights between 500 and $999 \mathrm{~g}$, and the mothers had education below the seven years between the ages of 15 and 24 . In relation to pregnancy, the period in which the greatest number of deaths was between 32 and 41 weeks, the incidence of cesarean section. Conclusion: Further

\footnotetext{
1 Enfermeira Especialista em Saúde Coletiva ênfase em Saúde da Família. Professora Substituta do Departamento de Enfermagem- UFSM. Integrante do grupo de pesquisa Cuidado à Saúde das Pessoas, Famílias e Sociedade- PEFAS. tassipotrich@yahoo.com.br

2 Acadêmica do curso de Nutrição do Centro Universitário Franciscano (UNIFRA), Santa Maria, RS. laissa_medeiros_1@hotmail.com

3 Acādêmica do curso de Nutrição do Centro Universitário Franciscano (UNIFRA), Santa Maria, RS. raquelpossobon@msn.com

4 Acadêmica do curso de Nutrição do Centro Universitário Franciscano (UNIFRA), Santa Maria, RS. paty_vianna@hotmail.com

${ }^{5}$ Nutricionista. Mestre em Ciência e Tecnologia Farmacêutica. Professora Assistente do Centro Universitário Franciscano (UNIFRA), Santa Maria, RS. ruth@yahoo.com.br

${ }^{6}$ Enfermeira pediatra. Professora Adjunta do Departamento e do Programa de pós-graduação em Enfermagem da Universidade Federal de Santa Maria. Membro do Grupo de Pesquisa Cuidado à Saúde das Pessoas, Famílias e Sociedade - PEFAS. elianeves03@gmail.com
} 


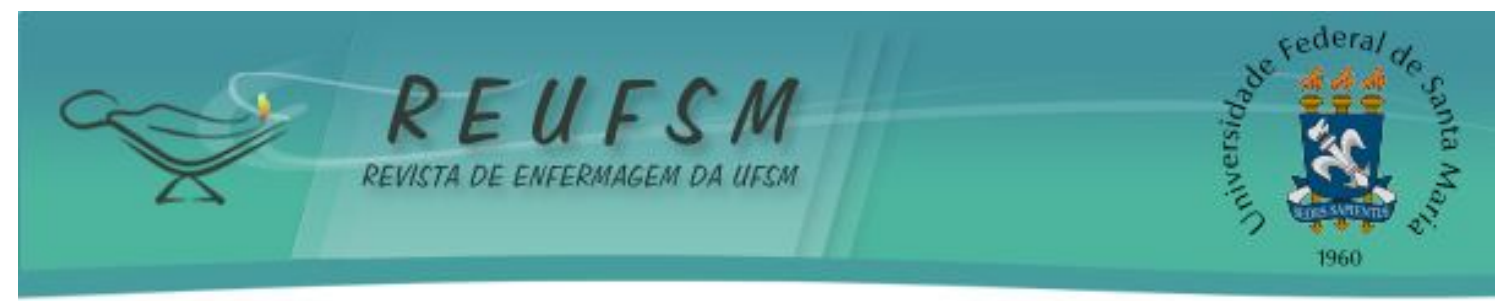

studies were recommended relating the findings with other risk factors, such as consultation and whether or not prenatal, maternal nutrition, number of pregnancies, in order to determine a reliable diagnosis of infant mortality in the municipality.

Descriptors: Infant mortality; Pregnancy; Birthweight.

RESUMEN: Objetivo: Analizar la mortalidad infantile de acuerdo con las características de la madre, el embarazo y el peso al nacer en la ciudad de Santa Maria, RS. Métodos: pesquisa cuantitava y descriptiva. Las informaciones fueron recogidas en el sitio del Ministeria de Salud, en el banco de datos del Departamento de Informática del Sistema Único de Salud/DATASUS en el período entre 2000 hasta 2008. Resultados: se observó una prevalencia más alta de mortalidad en niños con peso al nacer entre 500 y $999 \mathrm{~g}$, y las madres tenían educación inferior a los siete años entre las edades de 15 y 24 años. En relación con el embarazo, el período en que el mayor número de muertes fue entre 32 y 41 semana en la incidencia del parto por cesárea. Consideraciones finales: se recomienda más estudios sobre los resultados con otros factores de riesgo, como realización o no de consultas prenatales, la nutrición materna, el número de embarazos, con el fin de determinar un diagnóstico fiable de la mortalidad infantil en el municipio.

Descriptores: La mortalidad infantil; El embarazo; Peso al nacer.

\section{INTRODUÇÃO}

As condições de saúde de uma população podem ser retratadas através de indicadores de saúde construídos a partir de dados confiáveis gerados no seio da sociedade. ${ }^{1} \mathrm{~A}$ mortalidade infantil é classicamente considerada um dos melhores indicadores do nível de vida e bem estar social de uma população. ${ }^{2} 0$ Brasil ainda conta com níveis alarmantes e eticamente inaceitáveis de mortalidade infantil, apesar das taxas decrescentes nos últimos 20 anos. Estudos têm demonstrado a importância de intervenções na redução da mortalidade infantil em todo o País, com destaque especial para as ações dos serviços de saúde. ${ }^{3}$

Conforme o Relatório do Fundo das Nações Unidas para a Infância (UNICEF), os dados sobre mortalidade infantil, no Brasil, e seus componentes neonatal e pós-neonatal evidenciam, tanto a tendência decrescente desses indicadores quanto a sua heterogeneidade. ${ }^{4}$ Em 1991, o Coeficiente de Mortalidade Infantil (CMI), no Brasil, era de $45,2 \%$ caindo para $24,9 \%$ Nascidos Vivos (NV) em 2006. As regiões Nordeste e Norte do País apresentavam, respectivamente, $36,9 \%$ e $25,8 \%$ NV, enquanto as regiões CentroOeste, Sudeste e Sul portavam taxas de $19,5 \%$, 18,3\% e 16,7\% NV, respectivamente em 2006. Essa forma heterogênea, como se apresentam as taxas de mortalidade infantil em unidades federativas do Brasil, pode revelar distintas condições de saúde e assistência a que estão submetidos os brasileiros e as diferentes exposições ao óbito precoce no País.

Os dados de óbitos e nascimentos necessários para o cálculo da mortalidade infantil são originários de duas fontes principais: o Ministério da Saúde, através do Sistema de Informação de Mortalidade (SIM) e do Sistema de Informação sobre Nascidos Vivos (SINASC) e o Instituto Brasileiro de Geografia e Estatística (IBGE), responsável pelas estatísticas do registro civil. ${ }^{5}$

A mortalidade nos primeiros dias de vida expressa a complexa conjunção de fatores biológicos, socioeconômicos e assistenciais, sendo que estes últimos estão relacionados à atenção à gestante e ao recém-nascido. ${ }^{6}$ Dentre os fatores de risco para a ocorrência dos óbitos infantis evidenciados por diversos estudos, destaca-se o nascer com baixo peso, a prematuridade, o número de consultas de pré-natal igual ou menor do que seis, o índice de apgar, no $1^{0}$ e 50 minutos, inferior a sete, a gestação múltipla, os baixos níveis socioeconômico e de escolaridade materna, os recém-nascidos de mães solteiras e as precárias condições de saneamento e habitação. ${ }^{7-9}$ 


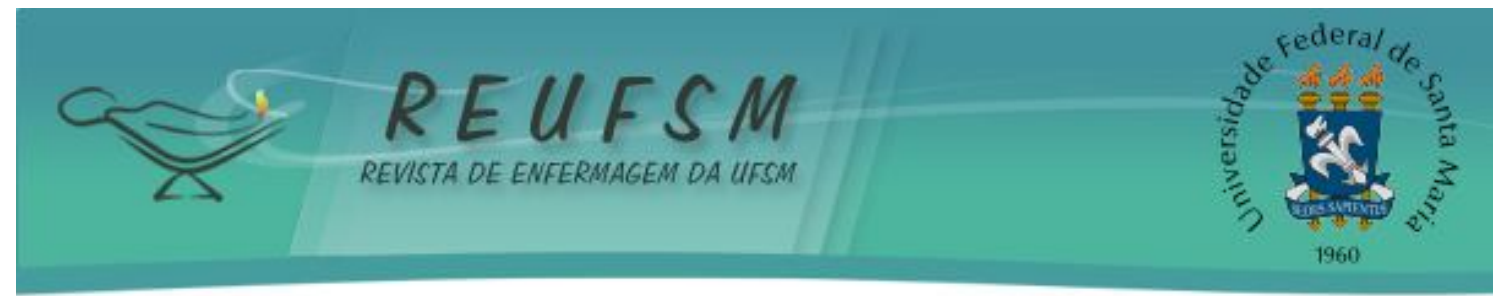

O número de filhos nascidos vivos é uma variável indicativa da distribuição dos filhos, segundo a sua ordem de nascimento e a idade da mãe, no momento do nascimento, associando-se ao nível socioeconômico da mãe e à mortalidade infantil. Alguns estudos mostram estreita relação entre múltiplas gestações e a mortalidade neonatal. ${ }^{10-11}$

Diante dos estudos citados acima e aliado às taxas de mortalidade infantil no município de Santa Maria surgiu a necessidade de identificar qual a prevalência de mortalidade infanfil associada a características da mãe, gestação e peso ao nascer. Neste contexto, o presente estudo teve como objetivo analisar a mortalidade infantil, segundo características da mãe, gestação e peso ao nascer na cidade de Santa Maria, RS.

\section{MÉTODO}

Trata-se de uma pesquisa quantitativa descritiva. As informações foram coletadas no site do Ministério da Saúde, com dados do município de Santa Maria-RS, no banco de dados do Departamento de Informática do Sistema Único de Saúde-DATASUS. Os dados foram extraídos do site e inseridos em tabelas no manuscrito, sendo que as variáveis foram analisadas apenas em freqüência absoluta.

Foram incluídos dados de mortalidade infantil relacionados às variáveis peso ao nascer, duração da gestação e características da mãe, tais como: escolaridade, tipo de parto e idade da mãe no período entre 2000 a 2008. Os dados foram analisados quantitativamente e apresentados sob forma de tabelas.

Tendo em vista que, os dados utilizados são provenientes de um site de acesso público, de acordo com o Comitê de Ética e Pesquisa da Universidade Federal de Santa Maria, o presente estudo não necessita passar por aprovação do mesmo.

\section{RESULTADOS E DISCUSSÃO}

No que diz respeito aos óbitos ocorridos, de acordo com o peso ao nascer, foram obtidos os dados abaixo descritos na tabela 1.

Tabela 1 - Distribuição de óbitos infantis, por ano, e segundo o peso ao nascer na cidade de Santa Maria- RS (2000-2008).

\begin{tabular}{cccccccccc}
\hline ANO & $\begin{array}{c}\text { Menos } \\
\mathbf{d e} \\
\mathbf{5 0 0 g}\end{array}$ & $\begin{array}{c}\mathbf{5 0 0} \mathbf{a} \\
\mathbf{9 9 9} \mathbf{g}\end{array}$ & $\mathbf{1 0 0 0} \mathbf{1 4 9 9} \mathbf{g}$ & $\mathbf{1 5 0 0} \mathbf{2 4 9 9} \mathbf{g}$ & $\mathbf{2 5 0 0} \mathbf{2 9 9 9} \mathbf{g}$ & $\begin{array}{c}\mathbf{3 0 0 0} \\
\mathbf{a} \\
\mathbf{3 9 9} \\
\mathbf{g}\end{array}$ & $\begin{array}{c}\mathbf{4 0 0 0 g} \\
\text { e mais }\end{array}$ & Ignorado & Total \\
\hline 2008 & 2 & 13 & 5 & 9 & 8 & 3 & - & - & 40 \\
2007 & 1 & 14 & 4 & 5 & 4 & 7 & - & - & 35 \\
2006 & 3 & 15 & 9 & 10 & 5 & 6 & 2 & - & 50 \\
2005 & 1 & 17 & 6 & 10 & 3 & 8 & 1 & - & 46 \\
2004 & - & 7 & 5 & 14 & 5 & 7 & 1 & 3 & 42 \\
2003 & 4 & 8 & 5 & 14 & 4 & 6 & 1 & 13 & 55 \\
2002 & - & 19 & 1 & 10 & 3 & 8 & 1 & 17 & 59 \\
2001 & 2 & 7 & 6 & 7 & 2 & 3 & - & 18 & 45 \\
2000 & 1 & 11 & 7 & 4 & 5 & 10 & - & 13 & 51 \\
\hline Total: & 14 & 111 & 48 & 83 & 58 & 58 & 6 & 64 & 423 \\
\hline
\end{tabular}

Fonte: DATASUS. 


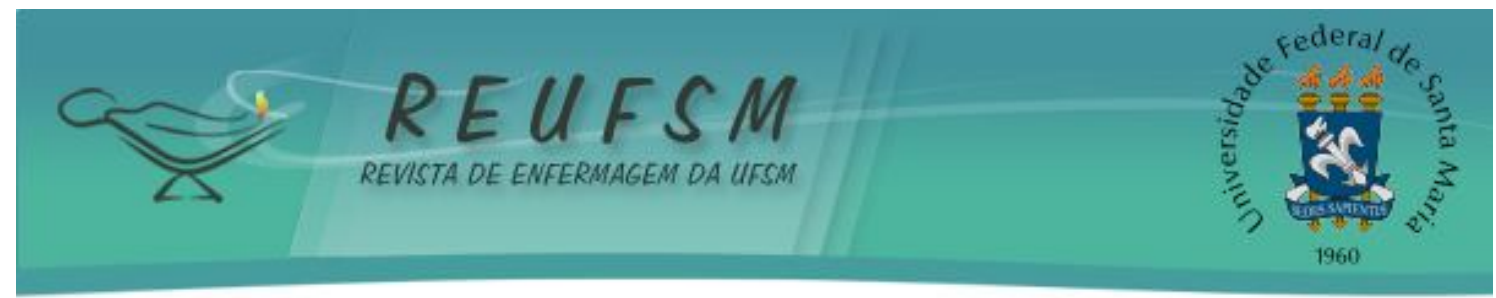

De acordo com a tabela 1, evidenciou-se que, no ano de 2002, ocorreram 59 óbitos, sendo que destes, 19, apresentavam peso entre 500 e $999 \mathrm{~g}$. Os dados mostram também que, no período avaliado, houve maior frequência de óbitos em RNs de 500 a 999g, exceto nos anos de 2003 e 2004. Observou-se, também, que crianças nascidas com $4.000 \mathrm{~g}$, ou mais, apresentaram um número reduzido de óbitos, um total de 6 .

Evidenciou-se também que, a partir de 2005, não houve nenhuma perda nos resultados, ou seja, todos os óbitos, ocorridos entre 2005 e 2008, encontravam-se com registros completos.

Em estudo realizado na maternidade do município de Campinas (SP), com recémnascidos, para identificar mortalidade, constatou-se que todos os nascidos com peso inferior a $1.000 \mathrm{~g}$ morreram. Um dos aspectos a ser considerado refere que a probabilidade de morte diminui, na medida em que aumenta o peso da criança ao nascer: o RecémNascido (RN) de muito baixo peso (menos de $1.500 \mathrm{~g}$ ) apresenta chance de falecer no período neonatal, 155 vezes maior que as crianças com 2.500 gramas ou mais. ${ }^{12-13}$

Ao analisar dados referentes à escolaridade das mães de crianças que foram a óbito neste período, tem-se a tabela a seguir:

Tabela 2 - Distribuição de óbitos infantis, por ano, e segundo a escolaridade da mãe na cidade de Santa Maria- RS (200-2008).

\begin{tabular}{ccccccccc}
\hline ANO & Nenhuma & $\begin{array}{c}\mathbf{1} \text { a } \mathbf{3} \\
\text { anos }\end{array}$ & $\begin{array}{c}\mathbf{4} \text { a } \mathbf{7} \\
\text { anos }\end{array}$ & $\begin{array}{c}\mathbf{8} \text { a } \mathbf{1 1} \\
\text { anos }\end{array}$ & $\begin{array}{c}\mathbf{1 2} \text { anos e } \\
\text { mais }\end{array}$ & $\mathbf{2 9}$ grau & Ignorado & Total \\
\hline 2008 & 4 & 2 & 8 & 13 & - & 7 & 6 & 40 \\
2007 & - & 1 & 7 & 17 & - & 4 & 6 & 35 \\
2006 & 1 & 3 & 9 & 19 & - & 9 & 9 & 50 \\
2005 & 1 & 2 & 15 & 8 & - & 7 & 13 & 46 \\
2004 & 2 & 2 & 10 & 13 & - & 8 & 7 & 42 \\
2003 & 2 & 7 & 11 & 10 & - & 5 & 20 & 55 \\
2002 & 3 & 7 & 23 & 6 & - & 5 & 15 & 59 \\
2001 & 2 & 2 & 14 & 7 & - & 6 & 14 & 45 \\
2000 & 4 & 3 & 16 & 7 & 2 & - & 19 & 51 \\
\hline Total: & 19 & 29 & 113 & 100 & 2 & 51 & 109 & 423 \\
\hline Fon
\end{tabular}

Fonte: DATASUS.

Outro dado relevante neste estudo foi que o nível de escolaridade da mãe pode estar relacionado ao número de óbito, pois mães que frequentaram a escola por, no máximo, sete anos (4 a 7 anos), ou seja, o ensino fundamental incompleto, a ocorrência de óbitos foi maior, apresentando 23 casos no ano de 2002 e um total de 113 óbitos durante todo o período analisado.

A maior taxa de mortalidade neonatal precoce foi encontrada entre RN de mães que declararam não possuir qualquer ano de estudo, valor 20 vezes maior do que aquele do grupo de RN de mães com maior escolaridade. ${ }^{14}$ Entende-se que a baixa escolaridade significa menor acesso à informação, determinando também menores condições de acesso aos serviços de saúde, e ainda, que a maior escolaridade na educação feminina, além do aprimoramento que proporciona à vida das mulheres, permite maior acesso às informações de saúde. ${ }^{15-16}$

Ao relacionar o tipo de parto com a ocorrência do óbito infantil, apresenta-se a seguinte tabela: 


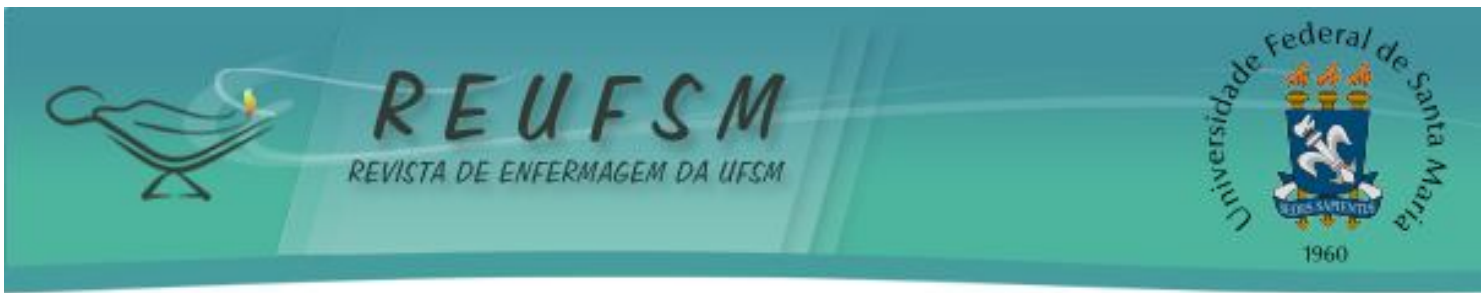

Tabela 3 - Distribuição de óbitos infantis, por ano, e segundo o tipo de parto na cidade de Santa Maria- RS (2000-2008).

\begin{tabular}{ccccc}
\hline Ano & Vaginal & Cesáreo & Ignorado & Total \\
\hline 2008 & 12 & 28 & - & 40 \\
2007 & 18 & 16 & 1 & 35 \\
2006 & 22 & 28 & - & 50 \\
2005 & 18 & 24 & 4 & 46 \\
2004 & 16 & 22 & 4 & 42 \\
2003 & 18 & 25 & 12 & 55 \\
2002 & 23 & 26 & 10 & 59 \\
2001 & 12 & 19 & 14 & 45 \\
2000 & 21 & 19 & 11 & 51 \\
\hline Total: & 160 & 207 & 56 & 423 \\
\hline
\end{tabular}

Fonte: DATASUS.

Quanto ao tipo de parto, a cesárea obteve um total de 207 óbitos no período analisado. As desvantagens para os recém-nascidos de parto cesáreo incluem prematuridade, baixo peso e consequências, como maior probabilidade de permanência em Unidades de Terapia Intensiva (UTI). ${ }^{16}$

Em um estudo com mães residentes em municípios-sede de Regionais de Saúde, no Paraná, constatou-se que a forma preferencial de parto foi a cesariana $(55 \%$, concordando com estudos que trazem taxas elevadas do mesmo tipo de parto e ressaltam, ainda, que a cesariana pode aumentar o tempo de permanência da mãe e do filho no hospital, a recuperação puerperal tardia e o consumo de medicamentos, os quais podem representar risco para a morbimortalidade materna e infantil. ${ }^{17-18}$

A tabela 4 mostra dados referentes à idade materna relacionada ao óbito infantil.

Tabela 4 - Distribuição de óbitos infantis, por ano, e segundo a idade materna na cidade de Santa Maria-RS (2000-2008).

\begin{tabular}{ccccccccccc}
\hline ANO & $\begin{array}{c}\mathbf{1 0} \mathbf{a} \\
\mathbf{1 4} \\
\text { anos }\end{array}$ & $\begin{array}{c}\mathbf{1 5} \mathbf{a} \\
\mathbf{1 9} \\
\text { anos }\end{array}$ & $\begin{array}{c}\mathbf{2 0} \mathbf{2 4} \\
\mathbf{a n o s}\end{array}$ & $\begin{array}{c}\mathbf{2 5} \mathbf{a} \\
\mathbf{2 9} \\
\text { anos }\end{array}$ & $\begin{array}{c}\mathbf{3 0} \mathbf{a} \\
\mathbf{3 4} \\
\text { anos }\end{array}$ & $\begin{array}{c}\mathbf{3 5} \mathbf{a} \\
\mathbf{3 9} \\
\text { anos }\end{array}$ & $\begin{array}{c}\mathbf{4 0} \mathbf{a} \\
\mathbf{4 4} \\
\text { anos }\end{array}$ & $\begin{array}{c}\mathbf{4 5} \mathbf{a} \\
\mathbf{4 9} \\
\text { anos }\end{array}$ & $\begin{array}{c}\text { Idade } \\
\text { ignorada }\end{array}$ & Total \\
\hline 2008 & 2 & 8 & 11 & 9 & 3 & 4 & 2 & - & 1 & 40 \\
2007 & - & 11 & 12 & 6 & 3 & 1 & 2 & - & - & 35 \\
2006 & 1 & 11 & 14 & 9 & 7 & 7 & 1 & - & - & 50 \\
2005 & - & 9 & 14 & 10 & 6 & 4 & 1 & 1 & 1 & 46 \\
2004 & - & 9 & 5 & 13 & 7 & 2 & 3 & - & 3 & 42 \\
2003 & 1 & 14 & 8 & 11 & 10 & 1 & 1 & - & 9 & 55 \\
2002 & 3 & 13 & 11 & 12 & 10 & 2 & 2 & - & 6 & 59 \\
2001 & - & 9 & 6 & 7 & 4 & 5 & 1 & - & 13 & 45 \\
2000 & 1 & 8 & 8 & 6 & 8 & 8 & 1 & - & 11 & 51 \\
\hline Total: & 8 & 92 & 89 & 83 & 58 & 34 & 14 & 1 & 44 & 423 \\
\hline
\end{tabular}

Fonte: DATASUS 


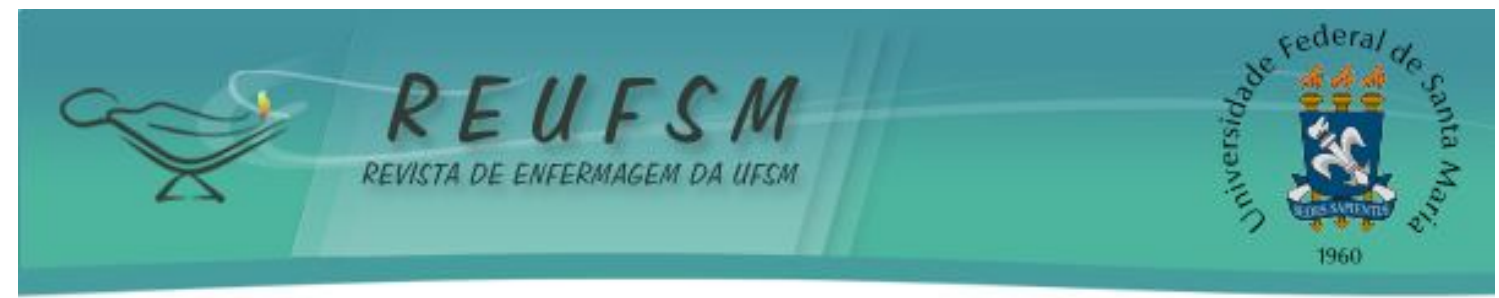

Dentre os dados obtidos na tabela 4, pode-se observar que ocorreram 92 óbitos, durante o período analisado, em filhos de mães adolescentes (dos 15 aos 19 anos), sendo que, na faixa etária de 20 a 24 anos, ocorreram 89 óbitos no mesmo período.

A idade materna é uma variável muito utilizada em estudos epidemiológicos, no entanto, a relação da idade da mãe e os índices de mortalidade neonatal não estão bem esclarecidos, pois os maiores índices de mortalidade não apresentaram diferença entre mães adolescentes e com mais de 35 anos. ${ }^{19}$ Outros estudos afirmam que essas variáveis são positivas, indicando que a idade influencia na mortalidade neonatal. ${ }^{7,20} \mathrm{~J}$ á, em um estudo, em Salvador, constatou-se o maior risco de morte neonatal precoce, apenas entre RN, de gestantes abaixo de 20 anos, entre os anos de 2000 a $2005 .^{20}$

Foram analisados, também, dados referentes à idade gestacional, sendo apresentados na tabela abaixo:

Tabela 5- Distribuição de óbitos infantis, por ano, e segundo a duração da gestação na cidade de Santa Maria- RS(2000-2008).

\begin{tabular}{|c|c|c|c|c|c|c|c|c|}
\hline Ano & $\begin{array}{c}\text { Menos de } \\
22 \\
\text { semanas }\end{array}$ & $\begin{array}{c}22 \text { a } 27 \\
\text { semanas }\end{array}$ & $\begin{array}{c}28 \text { a } 31 \\
\text { semanas }\end{array}$ & $\begin{array}{l}32 \text { a } 36 \\
\text { semanas }\end{array}$ & $\begin{array}{c}37 \text { a } 41 \\
\text { semanas }\end{array}$ & $\begin{array}{c}42 \\
\text { semanas e } \\
\text { mais }\end{array}$ & Ignorado & Total \\
\hline 2008 & 1 & 10 & 11 & 7 & 11 & - & - & 40 \\
\hline 2007 & 1 & 12 & 2 & 12 & 6 & - & 2 & 35 \\
\hline 2006 & 1 & 18 & 6 & 11 & 12 & - & 2 & 50 \\
\hline 2005 & 3 & 11 & 10 & 9 & 8 & 1 & 4 & 46 \\
\hline 2004 & - & 4 & 5 & 14 & 16 & - & 3 & 42 \\
\hline 2003 & 1 & 7 & 11 & 11 & 10 & 3 & 12 & 55 \\
\hline 2002 & - & 18 & 5 & 14 & 12 & 3 & 7 & 59 \\
\hline 2001 & 3 & 9 & 5 & 5 & 8 & 2 & 13 & 45 \\
\hline 2000 & 1 & 10 & 7 & 10 & 11 & 3 & 9 & 51 \\
\hline Total: & 11 & 99 & 62 & 93 & 94 & 12 & 52 & 423 \\
\hline
\end{tabular}

Fonte: DATASUS.

Os dados da Tabela 5 demonstram que, entre os anos de 2002 a 2006, ocorreram 18 óbitos com idade gestacional de 22 a 27 semanas. Evidenciou-se, também, que, em relação à duração da gestação, ocorreram 99 óbitos entre o período de 22 a 27 semanas.

Em relação à duração da gestação, foi apontado maior percentual de óbitos entre RN prematuros, isto é, de mães que pariram com 22 a 36 semanas de gestação. ${ }^{20} \mathrm{~A}$ comparação entre 92 neonatos, que faleceram antes de 168 horas e os sobreviventes, mostrou maior frequência, entre os óbitos, com idade gestacional de $23-27$ semanas. $^{21}$

A probabilidade de morte neonatal diminui, significativamente, à medida que aumenta a duração da gestação: entre os nascidos vivos pré-termo, a mortalidade neonatal mostra-se 28 vezes mais elevada que entre os nascidos vivos de gestações a termo. A criança pós-termo (42 semanas e mais), por sua vez, tem probabilidade de morte 1,7 vezes maior que aquela nascida a termo. ${ }^{13}$

\section{CONCLUSÃO}

O estudo possibilitou evidenciar algumas características referentes à mortalidade infantil do município de Santa Maria- RS, de acordo com dados do DATASUS. Ao avaliar a relação entre o peso ao nascer e a mortalidade, constatou-se que, na maioria dos casos, o óbito ocorreu em recém-nascidos com peso entre 500 e $999 \mathrm{~g}$, sendo que o menor número de óbitos ocorreu com recém-nascidos com peso igual ou superior a $4.000 \mathrm{~g}$. 


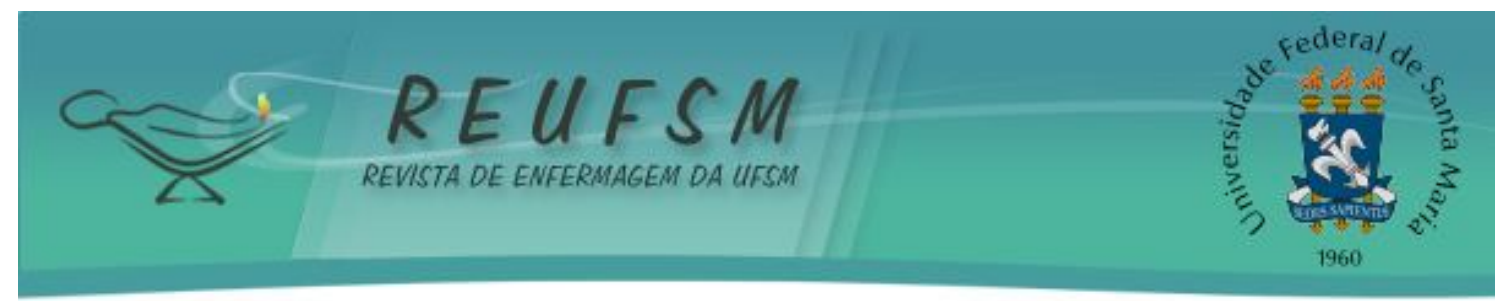

No que diz respeito à escolaridade, os óbitos foram mais frequentes nos casos em que a escolaridade da mãe não ultrapassou sete anos. 0 tipo de parto também pode ser relacionado à mortalidade infantil. Segundo o estudo, a ocorrência de óbitos foi mais frequente nos casos em que foi realizado o parto cesáreo.

Quanto à idade materna, identificou-se a maior ocorrência de casos com mães jovens em idade entre 15 e 24 anos. A relação entre idade materna e mortalidade infantil ainda não está bem esclarecida na literatura, pois vários estudos foram realizados, constatando-se resultados contraditórios. Sendo assim, torna-se necessária a realização de novos estudos que correlacionem outras variáveis em relação à gestação, para melhor identificação de fatores de risco.

Ao analisar a idade gestacional/duração da gestação, percebeu-se que a maior ocorrência de óbitos foi no período compreendido entre a $32^{a}$ e a $41^{a}$ semana de gestação. Este achado mostra-se contraditório ao que se encontra na literatura, necessitando-se, assim, de novos estudos referentes à relação entre a idade gestacional e a mortalidade infantil.

No que diz respeito ao tipo de parto, pôde-se evidenciar uma maior quantidade de óbitos em decorrência do parto cesáreo. Tal fato é evidenciado em diversos outros estudos, sendo que, mesmo apesar do conhecimento desse fator de risco, o parto cesáreo ainda é o mais comum.

Por fim, verificou-se que, na cidade Santa Maria, houve uma discreta diminuição da mortalidade infantil, ao longo dos oito anos pesquisados, porém, evidenciou-se um achado que foge do obj etivo da pesquisa, mas que julga-se relevante na avaliação dos indicadores de mortalidade infantil no Município, sendo ele a alta frequência de mortalidade infantil, ocorrida no ano de 2002.

Conhecer as características da população materno-infantil em seu município ou região é um dos principais requisitos que contribui para a eliminação dos riscos e o alcance de uma assistência de saúde com equidade. Sendo assim, recomenda-se um estudo objetivando avaliar os óbitos ocorridos no ano de 2002, no município de Santa Maria, a fim de avaliar, se a incidência destes deu-se, devido ao maior número de partos no ano ou a al guma variável não identificada.

Desse modo, recomendam-se novos estudos que relacionem os achados com outros fatores de risco, como a não realização de consultas pré-natais, a alimentação materna deficiente, o grande número de gestações, entre outros, para, assim, determinar um diagnóstico fidedigno da mortalidade infantil do município de Santa Maria. Diante disso, torna-se possível a formulação de ações que promovam a melhoria da qualidade de vida das gestantes, reduzindo as taxas de mortalidade infantil.

\section{REFERÊNCIAS}

1. Alves KCG, Zandonade E. Tendências da mortalidade infantil no Estado do Espírito Santo, Brasil, 1979 a 2004. Rev APS. 2009; 12(3): 302-310.

2. Andrade SM, Soares DA, Matsuo T, Souza RKT, Mathias TAF, Iwakura MLH et al. Condições de vida e mortalidade infantil no Estado do Paraná, Brasil, 1997-2001. Cad saúde pública. 2006; 22(1): 181-189.

3. Silva LMV, Paim J S, Costa MCN. Desigualdades na mortalidade, espaço e estratos sociais. Rev saúde pública. 1999; 33(2): 187-197.

4. Fundo das Nações Unidas para a Infância. Situação mundial da infância 2008: Caderno Brasil. Brasília; 2008. [acesso em: 26 mar 2011]. Disponível em: http:// www. unicef. org/ brazil/ sib06h. htm.

5. Duarte CMR. Reflexos das políticas de saúde sobre as tendências da mortalidade infantil no Brasil: revisão da literatura sobre a última década. Cad saúde pública. 2007; 23(7): 1511-1528.

6. Drumond EF, Machado CJ, França E. Óbitos neonatais precoces: análise de causas múltiplas de morte pelo método Grade of Membership. Cad saúde pública. 2007; 23(1): 157-166. 


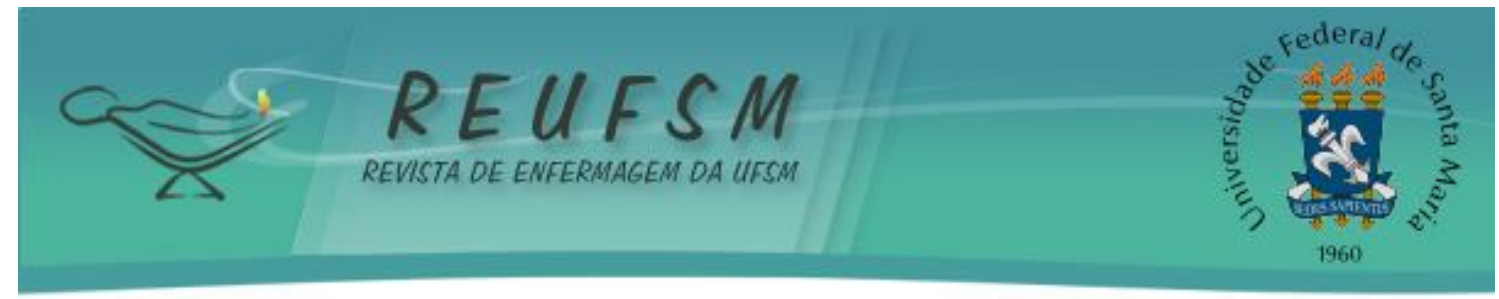

7. Giglio MRP, Lamounier JA, Neto OLM, César CC. Baixo peso ao nascer em coorte de recém-nascidos em Goiânia-Brasil no ano de 2000. Rev bras ginecol obstet. 2005; 27(3): 130-136.

8. Leal MC, Gama SGN, Campos MR, Cavalini LT, Garbayo LS, Brasil CLP et al. Fatores associados à morbimortalidade perinatal em uma amostra de maternidades públicas e privadas do município do Rio de J aneiro, 1999-2001. Cad saúde pública. 2004; 20(1): S20S33.

9. Jobim R, Aerts D. Mortalidade infantil evitável e fatores associados em Porto Alegre, Rio Grande do Sul, Brasil, 2000-2003. Cad saúde pública. 2008; 24(1): 179-187.

10. Carvalho M, Gomes MA. A mortalidade do prematuro extremo em nosso meio: realidade e desafios. J pediatr. 2005; 81:111-118.

11. Ferrari LSL, Brito ASJ, Carvalho ABR, Gonzáles MRC. Mortalidade neonatal no município de Londrina, Paraná, Brasil, nos anos de 1994, 1999 e 2002. Cad saúde pública. 2006; 22(1): 1.063-1.071.

12. Mariotini GGB, Filho AAB. Peso ao nascer e mortalidade hospitalar entre nascidos vivos, 1975-1996. Rev saúde pública. 2000; 34(1): 71-76.

13. Ortiz LP, Oushiro DA. Perfil da mortalidade neonatal no Estado de São Paulo. São Paulo Perspectiva. 2008; 22(1): 19-29.

14. Soares ES, Menezes GMS. Fatores associados à mortalidade neonatal precoce: análise de situação no nível local. Epidemiologia Serviços de Saúde. 2010; 19(1): 51-60.

15. Coimbra LC, Silva AAM, Mochel EG, Alves MTSSB, Ribeiro VS, Aragão VMF, et al. Fatores associados à inadequação do uso da assistência pré-natal. Cad saúde pública. 2003; 37(4): 456-62.

16. Uchimura TT, Pelissarii DM, Soares DFPP, Uchimura NS, Santana RG, Moraes CMS. Fatores de risco para o baixo peso ao nascer, segundo as variáveis da mãe e do recémnascido, em Maringá - PR, no período de 1996 a 2002. Ciênc cuid saúde. 2007; 6(1): 51-8.

17. Mello EC, Mathias TAF. Características das mães e dos recém-nascidos em municípios-sede de regional de saúde, no Paraná, a partir dos dados do SINASC. Rev Cogitare enferm. 2010; 15(2): 293-301.

18. Souza JJC, Kunkel N, Gomes MA, Freitas PF. Equidade inversa e desigualdades no acesso à tecnologia no parto em Santa Catarina, Brasil, 2000 a 2004. Rev Bras Saúde Mater Infant. 2007; 7(4): 397-403.

19. César CC, Ribeiro PM, Abreu DMX. Efeito-idade ou efeito-pobreza? Mães adolescentes e mortalidade neonatal em Belo Horizonte. Rev Bras Est Pop. 2000; 17: 177-196.

20. Almeida MF, Novaes HMD, Alencar GP, Rodrigues LC. Mortalidade neonatal no município de São Paulo: influência do peso ao nascer e de fatores sociodemográficos e assistenciais. Rev bras epidemiol. 2002; 5(1): 93-107.

21. Almeida MFB, Guinsburg R, Martinez FE, Procianoy RS, Leone CR, Marba STM et al. Fatores perinatais associados ao óbito precoce em prematuros nascidos nos centros da Rede Brasileira de Pesquisas Neonatais. Arch Pediatr Urug. 2010; 81(2): 112-120.

Data de recebimento: 29/07/2011

Data de aceite: 11/ 10/2011

Contato com autora responsável: Tassiana Potrich

Endereço: Rua Appel, 132- Apto 04, Centro - Santa Maria - RS.

Cep: 97015030

E-mail: tassipotrich@yahoo.com.br 